\title{
Successful treatment of Lambert-Eaton myasthenic syndrome in a small cell lung cancer patient using 3,4-diaminopyridine: A case report
}

\author{
JAE-WON HYUN ${ }^{1}$, SU-HYUN KIM ${ }^{1}$, IN HYE JEONG ${ }^{1}$, HEUNG TAE KIM ${ }^{2}$ and HO JIN KIM ${ }^{1}$ \\ ${ }^{1}$ Department of Neurology; ${ }^{2}$ Center for Lung Cancer, Research Institute and Hospital of National Cancer Center, \\ Goyang, Gyeonggi 410-769, Republic of Korea
}

Received February 25, 2015; Accepted December 2, 2015

DOI: $10.3892 / 01.2016 .4123$

\begin{abstract}
Lambert-Eaton myasthenic syndrome (LEMS) is an autoimmune disorder of the neuromuscular junction that is characterized by muscle weakness. LEMS is usually associated with cancer, most commonly small cell lung cancer (SCLC). The potassium-channel blocker 3,4-diaminopyridine (3,4-DAP), has been previously used for the symptomatic treatment of LEMS. 3,4-DAP increases the release of acetylcholine and prolongs the duration of nerve action potentials at the presynaptic membrane of the neuromuscular junction. The present study describes the case of a patient with LEMS and SCLC, for which the symptoms did not improve with anticancer therapy, but did so markedly following treatment with 3,4-DAP. The present study illustrates that 3,4-DAP is a useful treatment choice in patients with LEMS, particularly for patients who do not fully improve following anticancer therapy.
\end{abstract}

\section{Introduction}

Lambert-Eaton myasthenic syndrome (LEMS) is an autoimmune disorder of neuromuscular transmission with presynaptic involvement (1). The syndrome exhibits characteristic proximal limb weakness, autonomic disturbance and depressed tendon reflexes. LEMS is a rare disease with an estimated annual incidence of $0.48-0.75$ cases per million individuals in the Netherlands, and a prevalence of 3.42 cases per million individuals (2). Diagnosis of LEMS is based on clinical symptoms and electrophysiological studies, including the repetitive nerve stimulation test (2). LEMS is associated with cancer in the form of paraneoplastic syndrome in $50-60 \%$ of cases, the majority of which are small cell lung cancer (SCLC) (3). Treatment is based on anti-cancer therapy

Correspondence to: Dr Ho Jin Kim, Department of Neurology, Research Institute and Hospital of National Cancer Center, 323 Ilsan Road, Goyang, Gyeonggi 410-769, Republic of Korea E-mail: hojinkim@ncc.re.kr

Key words: Lambert-Eaton myasthenic syndrome, small cell lung cancer, 3,4-diaminopyridine for those patients with associated cancers, given in combined with symptomatic and immunosuppressive therapy (4). LEMS patients with SCLC are more likely to reveal a better prognosis than patients with SCLC alone, probably due to early identification of SCLC (5). The potassium-channel blocker, 3,4-diaminopyridine (3,4-DAP), prolongs the duration of nerve action potentials and increases the release of acetylcholine at the presynaptic membrane of the neuromuscular junction, effectively treating the symptoms of LEMS (6-8). The present study describes the case of a patient with LEMS and SCLC, who showed a marked clinical improvement following treatment with 3,4-DAP, compared with anticancer therapy alone, which only caused temporary improvement. The patient provided written informed consent.

\section{Case report}

A 62-year-old male presented to the Hallym University Hospital (Chuncheon, Republic of Korea) in December 2013 with progressive quadriparesis, which had been apparent for $>2$ weeks and was more prominent in the legs compared with the arms. A diagnosis of myasthenia gravis (MG) was suspected initially due to progressive quadriparesis, as described above. In addition, thymoma was also suspected due to its high proportion of comorbidity with MG. The patient underwent a chest computed tomography (CT) scan to evaluate a thymoma. A subcarinal mass was subsequently detected. A bronchoscopic biopsy revealed a malignant small round cell tumor that was suggestive of small cell carcinoma, and the patient was diagnosed with SCLC. The patient visited the Research Institute and Hospital of the National Cancer Center (Goyang, Republic of Korea) for additional evaluation in January 2014. The patient experienced progressive weakness and fatigue, which appeared to be worse in the evening. The patient also complained of a dry mouth and mild dysarthria, with intermittent aspiration of water. The family history was normal, and the patient had a history of smoking one packet of cigarettes per day, for 38 years. A neurological examination revealed overall proximal weakness [Medical Research Council (MRC) grade IV+] and hypoactive tendon reflexes with normal sensory function (9). Since a diagnosis of LEMS was suspected, a repetitive nerve stimulation 
A

a

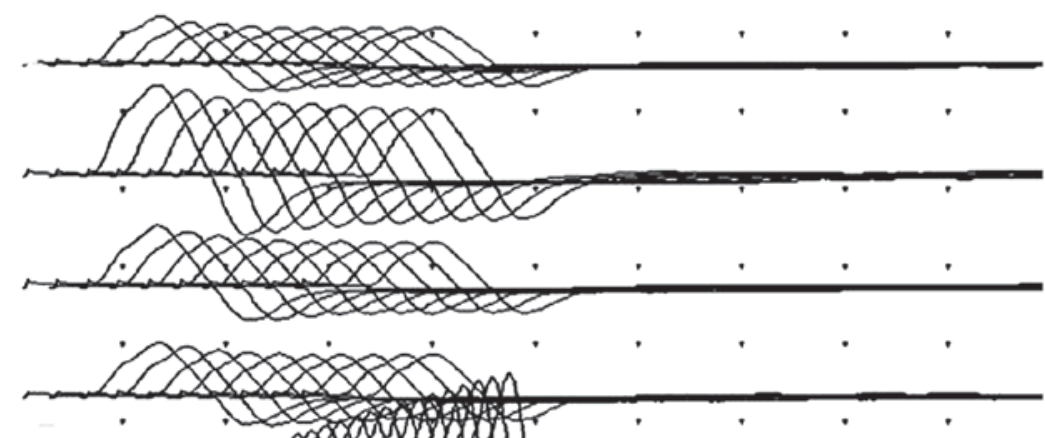

b

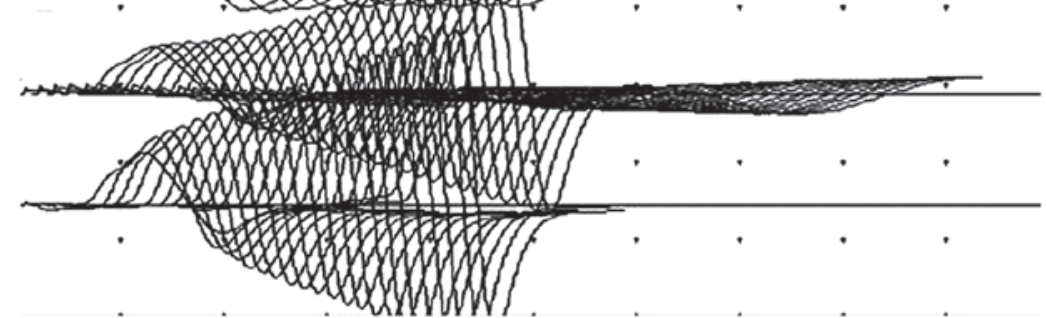

B $\mathbf{a}$

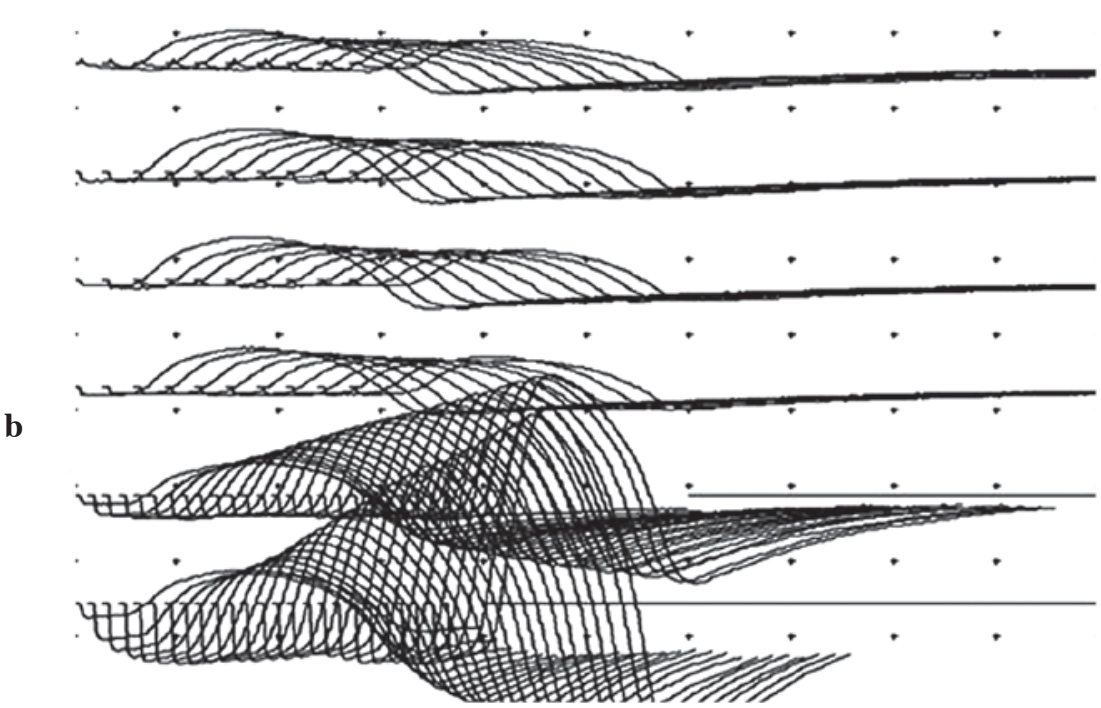

Figure 1. Repetitive nerve stimulation test. (A) Tests performed in abductor digiti minimi muscle (Aa) Abnormal decremental responses with low-rate stimulation. (Ab) Incremental responses with high-rate stimulation. (B) Tests performed in trapezius muscle. (Ba) Abnormal decremental responses with low-rate stimulation. (Bb) Incremental responses with high-rate stimulation.

test was performed on the right abductor digiti minimi, trapezius and orbicularis oculi muscles using the method previously described by $\mathrm{Oh}$ (10). The results were compatible with LEMS (Fig. 1). Abnormal decremental responses with low-rate stimulation and incremental responses with high-rate stimulation were observed. An anti-acetylcholine receptor antibody assay yielded a negative result.

Four cycles of chemotherapy (etoposide and carboplatin) and concurrent thoracic irradiation $(6,380 \mathrm{cGy} / 29 \mathrm{Fx})$ were performed, which resulted in a modest response of the SCLC (partial remission status). The MRC grading system divides muscle strength into 3 levels in grade IV. GIV+ means definite but slight weakness, GIV means able to move the joint against a combination of gravity and some resistance, and GIV- means capable of minimal resistance. Subsequent to the treatment for cancer, a mild improvement in the muscle weakness (MRC grade IV+) was recognized. However, the muscle weakness of the patient worsened again (MRC grade III to IV-) 1 month after the concurrent chemoradiation therapy (CCRT). The patient was initially treated with pyridostigmine alone $(60 \mathrm{mg}$, 3 times per day), as 3,4-DAP is an orphan drug not easily available in South Korea, but the symptoms remained unimproved for 2 weeks. Therefore, the patient was prescribed 3,4-DAP (10 mg, 3 times per day) from the Korean Orphan Drug Center (Seoul, South Korea) and pyridostigmine (60 mg, 3 times per day). The symptoms markedly improved following the administration of 3,4-DAP. A neurological examination performed 1 year later demonstrated no muscle weakness (MRC grade V) and no significant adverse effects were indicated.

\section{Discussion}

Previous studies in South Korea have reported a favorable response of LEMS to anticancer therapy that is used to treat SCLCalone (11-13). Specific anticancer therapies often improve LEMS, possibly by inhibiting the tumor-derived antigenic 
stimuli, including surface voltage-gated calcium channels (VGCCs), which provoke the production of anti-VGCC antibodies (14). By contrast, the patient in the present study experienced temporarily improved muscle weakness that then worsened, even with anticancer therapy. However, the patient showed a marked clinical improvement following the administration of 3,4-DAP. A number of previous studies in United States and Japan have demonstrated that patients with LEMS, who were treated with 3,4-DAP therapy exhibited significant improvements in muscle strength $(8,15,16)$. In accordance with the previous studies, the patient was diagnosed with muscle weakness caused by LEMS with SCLC, which was improved using 3,4-DAP therapy combined with CCRT. To the best of our knowledge, the present study is the first to report this finding in South Korea.

Aminopyridines potentiate chemical transmission at the central and peripheral synapses by targeting the VGCC and facilitating the release of acetylcholine at the motor nerve terminals. The chemical 3,4-DAP, at doses sufficient to improve neuromuscular transmission, produces few central nervous system adverse effects as its penetration into the brain is limited (6). However, epileptic seizures and arrhythmias have been previously described in patients that use high doses $(6,7)$.

The present study illustrates that 3,4-DAP is a useful treatment choice in patients with LEMS, particularly in patients that do not improve fully following anticancer therapy.

\section{References}

1. Lambert EH and Elmqvist D: Quantal components of end-plate potentials in the myasthenic syndrome. Ann NY Acad Sci 183 183-199, 1971.

2. Titulaer MJ, Lang B and Verschuuren JJ: Lambert-Eaton myasthenic syndrome: From clinical characteristics to therapeutic strategies. Lancet Neurol 10: 1098-1107, 2011.

3. O'Neill JH, Murray NM and Newsom-Davis J: The Lambert-Eaton myasthenic syndrome. A review of 50 cases. Brain 111: 577-596, 1988.

4. Maddison P. Treatment in Lambert-Eaton myasthenic syndrome. Ann NY Acad Sci 1275:78-84, 2012.
5. Titulaer MJ, Wirtz PW, Willems LN, van Kralingen KW, Smitt PA and Verschuuren JJ: Screening for small-cell lung cancer: A follow-up study of patients with Lambert-Eaton myasthenic syndrome. J Clin Oncol 26: 4276-4281, 2008.

6. Thomsen RH and Wilson DF: Effects of 4-aminopyridine and 3,4-diaminopyridine on transmitter release at the neuromuscular junction. J Pharmacol Exp Ther 227: 260-265, 1983.

7. McEvoy KM, Windebank AJ, Daube JR and Low PA: 3,4-Diaminopyridine in the treatment of Lambert-Eaton myasthenic syndrome. N Engl J Med 321: 1567-1571, 1989.

8. Sanders DB, Massey JM, Sanders LL and Edwards LJ: A randomized trial of 3,4-diaminopyridine in Lambert-Eaton myasthenic syndrome. Neurology 54: 603-607, 2000.

9. Medical Research Council: Aids to the examination of the peripheral nervous system. Memorandum No. 45. Her majesty's stationery office, London, UK, 1967.

10. Oh SJ: Repetitive Nerve Stimulation Test Principles of Clinical Electromyography: Case studies. 1st edition. Williams \& Wilkins, Baltimore, MD, USA, pp59-75, 1998.

11. Lee JH, Shin HY, Kim SM and Sunwoo IN: A case of lambert-eaton myasthenic syndrome with small-cell lung cancer and transient increase in anti-acetylcholine-receptor-binding antibody titer. J Clin Neurol 8: 305-307, 2012.

12. Shin DS, Park HY and Cho KH: Marked improvement of Lambert-Eaton syndrome with the chemotherapy of small cell lung carcinoma. J Korean Neurol Assoc 24: 95-97, 2006.

13. Lee SA, Sunwoo IN, Ro JK and Park KD: A case of remitted Lambert-Eaton Myathenic syndrome with small cell lung carcinoma following chemotherapy and radiotherapy. J Korean Neurol Assoc 8: 393-400, 1990.

14. Chalk CH, Murray NM, Newsom-Davis J, O'Neill JH and Spiro SG: Response of the Lambert-Eaton myasthenic syndrome to treatment of associated small-cell lung carcinoma. Neurology 40: 1552-1556, 1990.

15. Oh SJ, Claussen GG, Hatanaka $Y$ and Morgan MB: 3,4-Diaminopyridine is more effective than placebo in a randomized, double-blind, cross-over drug study in LEMS. Muscle Nerve 40: 795-800, 2009.

16. Uekita K, Ishida T, Sekine S, Ishii T, Sato S, Sugawara A, Tachihara M, Watanabe K, Kanazawa K, Saito J, et al: A case of Lambert-Eaton myasthenic syndrome with small cell lung cancer, treated with 3,4-diaminopyridine. Nihon Kokyuki Gakkai Zasshi 47: 76-80, 2009. 